\title{
Charge Noise in Organic Electrochemical Transistors
}

\author{
Ralph L. Stoop and Kishan Thodkar \\ Department of Physics, University of Basel, 4056 Basel, Switzerland \\ Michele Sessolo* and Henk J. Bolink \\ Instituto de Ciencia Molecular, Universidad de Valencia, 46980 Paterna, Spain \\ Christian Schönenberger \\ Department of Physics, University of Basel, 4056 Basel, Switzerland and Swiss Nanoscience Institute, \\ University of Basel, 4056 Basel, Switzerland \\ Michel Calame \\ Department of Physics, University of Basel, 4056 Basel, Switzerland; Swiss Nanoscience Institute, \\ University of Basel, 4056 Basel, Switzerland; and Swiss Federal Laboratories for Materials Science \\ and Technology (EMPA), 8600 Dübendorf, Switzerland \\ (Received 8 July 2016; revised manuscript received 18 November 2016; published 12 January 2017)

\begin{abstract}
Organic electrochemical transistors (OECTs) are increasingly studied as transducers in sensing applications. While much emphasis has been placed on analyzing and maximizing the OECT signal, noise has been mostly ignored, although it determines the resolution of the sensor. The major contribution to the noise in sensing devices is the $1 / f$ noise, dominant at low frequency. In this work, we demonstrate that the $1 / f$ noise in OECTs follows a charge-noise model, which reveals that the noise is due to charge fluctuations in proximity or within the bulk of the channel material. We present the noise scaling behavior with gate voltage, channel dimensions, and polymer thickness. Our results suggest the use of large area channels in order to maximize the signal-to-noise ratio (SNR) for biochemical and electrostatic sensing applications. A comparison with the literature shows that the magnitude of the noise in OECTs is similar to that observed in graphene transistors, and only slightly higher than that found in carbon nanotubes and silicon nanowire devices. In a model ion-sensing experiment with OECTs, we estimate crucial parameters such as the characteristic SNR and the corresponding limit of detection.
\end{abstract}

DOI: 10.1103/PhysRevApplied.7.014009

\section{INTRODUCTION}

The development of organic electronic devices has seen spectacular advances in recent decades, with the main driver being organic light-emitting diodes, which are now produced on an industrial scale [1]. Besides the ongoing, extensive research on organic solar cells and field-effect transistors (OFETs), the recent development of biosensors and bioelectronic devices based on conducting polymers has renewed attention towards organic electronic materials $[2,3]$.

These alternative applications stem from the advantages that conducting polymers offer when used as active elements in biosensing: (i) low-temperature solution processing, (ii) the possibility of coating large and flexible substrates, and (iii) the unique mixed electronic-ionic conductivity [4]. The final property is of particular interest for biochemical and electrophysiological sensing since ion exchange with the liquid environment lowers the

*Corresponding author. michele.sessolo@uv.es impedance at the electrolyte-polymer interface, enhancing the signal transduction [5].

A device type which has been intensively studied for applications in aqueous media is the organic electrochemical transistor (OECT) [6]. OECTs make use of hydrated conducting polymers which can change their conductivity by reversibly exchanging ions with an electrolyte. These processes take places at very low voltage $(<1 \mathrm{~V})$ and, since the current modulation is proportional to the amount of charge injected into the polymer channel, high transconductance $\left(g_{m}\right)$ can be achieved [7-9]. Since this working mechanism is especially favorable for a wide variety of sensing principles, OECTs have been implemented as enzymatic [10-12] and ion sensors [13], and they have been used both in vitro $[14,15]$ and in vivo $[7,16]$ to monitor biological [17] and electrophysiological [18] processes.

While much emphasis has been placed on analyzing and maximizing the OECT signal, noise has been mostly ignored. This lack of attention is very surprising, as noise is a key factor in determining the resolution of a sensor. When using a transistor as a biochemical sensor, the detection principle is based on the additional gating 

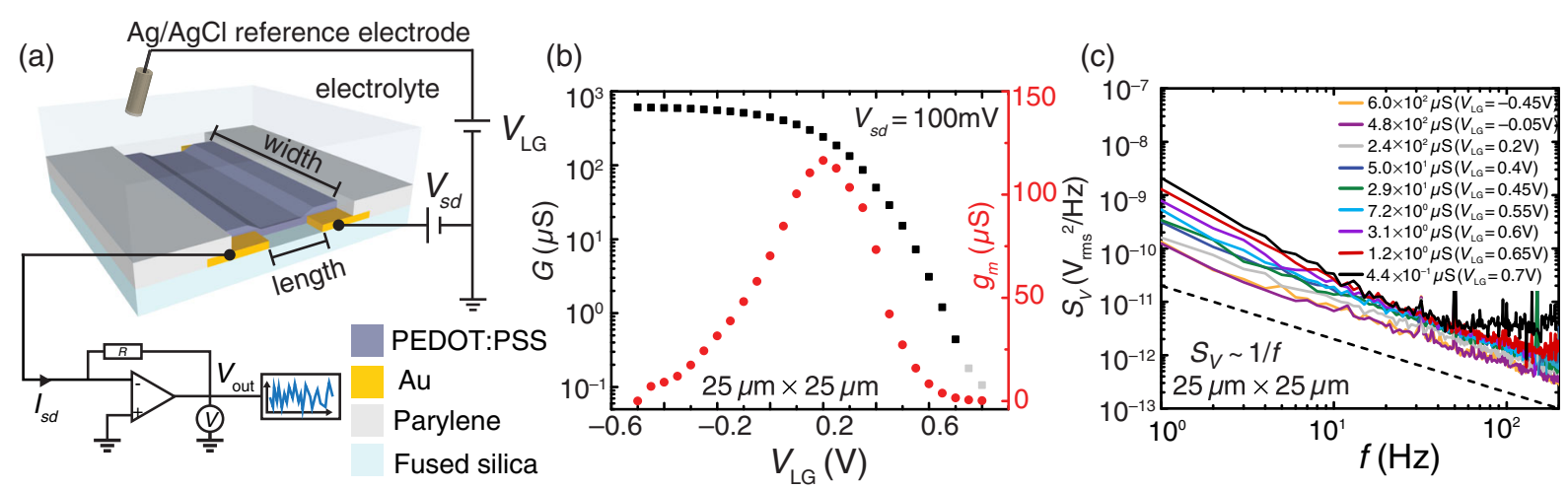

FIG. 1. (a) Device schematic and measurement setup for the noise characterization. (b) Conductance $G$ (black squares, left axis) and transconductance $g_{m}$ (red circles, right axis) versus the liquid-gate potential $V_{\mathrm{LG}}$ measured for a $25 \mu \mathrm{m}$ (width) $\times 25 \mu \mathrm{m}$ (length) OECT. (c) Power spectral density of the voltage fluctuations $S_{V}$ versus frequency $f$ for the OECT in (b) gated to the conductance values specified in the legend. The black dashed line indicates a $1 / f$ dependence. The majority of the noise spectra exhibit a $1 / f^{\alpha}$ characteristic, with $\alpha \approx 1$ and only a few exceptions showing $\alpha \approx 1.5$ in the low-frequency range. The scattering peaks are attributable to the intrinsic noise of the power line matching $50 \mathrm{~Hz}$ and multiples of it.

induced by charges adsorbed on the sensor surface. In this case, the noise determines the minimum detectable change of the gate voltage and can be related to the corresponding limit of detection (LOD) in terms of analyte concentration. The major contribution to the noise in sensing devices is the $1 / f$ noise, which is dominant at low frequency $[19,20]$. In biosensing, since the binding kinetics of many species of interest (e.g., proteins) typically require time scales up to a few minutes [21], the $1 / f$ noise becomes a key parameter limiting the performance of the sensor. For this reason, lowfrequency noise has been studied in depth for several transistor-based biosensors, such as silicon nanowires (SiNWs) operated as ion-sensitive field-effect transistors $[20,22,23]$, diamond solution-gated field-effect transistors [24], liquid-gated graphene $[19,25,26]$, and single-walled carbon-nanotube (SWCNT) transistors [27,28]. While some studies on OFETs exist [29,30], to the best of our knowledge, no measurements have been reported so far for OECTs.

In this work, we investigate the low-frequency noise of OECTs employing the conducting polymer poly(3,4-ethylenedioxythiophene) doped with poly(styrene sulfonic acid) (PEDOT:PSS). We present the noise scaling behavior with gate voltage, channel dimensions, and polymer thickness. We demonstrate that the noise in OECTs follows the predictions of the charge-noise model [19,27,31] (similar to the "background charge noise" found in single-electron transistor electrometers measured at low temperatures $[32,33])$, which scales inversely with the area of the channel. These results suggest the use of large-area PEDOT:PSS thin films in order to maximize the signalto-noise ratio (SNR) for biochemical and electrostatic sensing applications. No significant dependence of the noise on the polymer thickness is found. A comparison with the literature shows that the magnitude of the noise in PEDOT:PSS-based OECTs is similar to that observed in substrate-bound graphene transistors, and only slightly higher when compared to substrate-bound SWCNTs and SiNW devices. Finally, we estimate the characteristic SNR and the corresponding LOD of an OECT in a typical ionsensing measurement.

\section{MATERIALS AND METHODS}

Arrays of PEDOT:PSS OECTs with channel dimensions (width $\times$ length, $w \times l$ ) ranging from $5 \times 5 \mu \mathrm{m}$ to $250 \times$ $250 \mu \mathrm{m}$ and thickness $d$ from 60 to $800 \mathrm{~nm}$ are fabricated following a previously published protocol [7,34]. For devices with smaller dimensions $(1 \times 1 \mu \mathrm{m})$, an alternative process has been developed as described in the Supplemental Material [35]. Figure 1(a) depicts the device layout and the noise measurement setup. A liquid-gate potential $V_{\mathrm{LG}}$ is applied to a $\mathrm{Ag} / \mathrm{AgCl}$ reference electrode immersed in a $100-\mathrm{m} M \mathrm{KCl}$ aqueous solution to adjust the conductance of the PEDOT:PSS channel. Throughout this work, a constant source-drain voltage $V_{s d}=100 \mathrm{mV}$ is applied to bias the transistor. At each gate potential, the time-dependent source-drain current $I_{s d}(t)$ is measured using a current-voltage amplifier and transformed into a noise power spectral density $S_{I_{s d}}(f)$ (here referred to as current noise) via fast Fourier transformation where $t$ is the time and $f$ is the frequency [20]. The background noise measured at $V_{s d}=0 \mathrm{~V}$ is subtracted from the $1 / f$ spectrum measured at $V_{s d}=100 \mathrm{mV}$.

\section{RESULTS AND DISCUSSION}

\section{A. $\alpha$ noise versus charge noise}

Figure 1(b) shows a typical transfer characteristic (conductance $G$ and transconductance $g_{m}$ versus liquid-gate potential $\left.V_{\mathrm{LG}}\right)$ at $V_{s d}=100 \mathrm{mV}$ for a $(25 \times 25)-\mu \mathrm{m}$ OECT $(d \approx 110 \mathrm{~nm})$. The transconductance is defined here as 


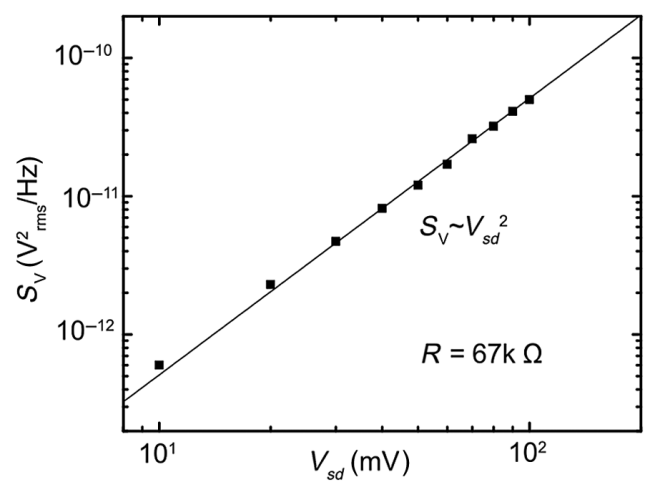

FIG. 2. Voltage noise $S_{V}=S_{V}(f=10 \mathrm{~Hz})$ versus sourcedrain voltage $V_{s d}$ for a $(25 \times 25)-\mu \mathrm{m}$ (thickness $\left.d \approx 110 \mathrm{~nm}\right)$ OECT gated to a resistance value of $R=67 \mathrm{k} \Omega$.

$g_{m}=\Delta I_{s d} / \Delta V_{\mathrm{LG}}$. The PEDOT:PSS film is highly conductive at zero applied gate voltage $\left(V_{\mathrm{LG}}=0 \mathrm{~V}\right)$ due to the intrinsic doping by PSS [8]. With an increasing positive $V_{\mathrm{LG}}$, potassium cations $\left(\mathrm{K}^{+}\right)$from the electrolyte enter the organic film, partially compensating the pendant sulphonate anions of the PSS and effectively decreasing the conductance [36]. The maximum transconductance is about $120 \mu \mathrm{S}$; when normalized with the source-drain voltage, this figure yields a value of approximately $1 \mathrm{mS} / \mathrm{V}$, as observed for state-of-the-art OECTs [7]. For each gate potential applied, the current noise $S_{I_{s d}}(f)$ is calculated. Hence, the corresponding voltage noise power spectral density can be obtained via $S_{V}(f)=S_{I_{s d}}(f) R^{2}$, where $R=$ $1 / G$ is the channel resistance. $S_{V}(f)$ is the voltage noise when the transistor is current biased and is commonly used to compare the noise of a transistor adjusted to different resistance values via the gate [20,37-39]. Figure 1(c) shows the voltage noise $S_{V}(f)$ of the $(25 \times 25)-\mu$ m channel transistor shown in Fig. 1(b) at nine different gate voltages. The spectrum shows a clear $1 / f^{\alpha}$ characteristic with $\alpha \approx 1$ in the low-frequency range, indicating that there are no single dominant processes taking place at a specific time scale [37]. For gate voltages higher than 0.7-1.0 V [gray highlighted points in Fig. 1(b)], leakage currents from the gate reference electrode to the source and drain contact start to be dominant and, therefore, determine the measured current. In terms of noise, the leakage currents lead to a dominant, frequency-independent noise contribution, which is not of the $1 / f$ type and is therefore not further analyzed for the noise studies.

It is commonly accepted that $1 / f$ noise is caused by resistance fluctuations. This type of noise is well described by Hooge's phenomenological law,

$$
\frac{S_{V}}{V_{s d}^{2}}=\frac{S_{I_{s d}}}{I_{s d}^{2}}=\frac{C}{f}=\frac{\alpha}{f N},
$$

where $C$ is a quantity of the noise of the sample and is constant for an Ohmic sample with constant resistance $R$.
Hooge redefined $C$ using the number of fluctuators $N$ and the Hooge parameter $\alpha$ : $C=\alpha / N$. Hooge's law does not give any insight into the physical meaning of $N$. The reason for its introduction was the idea that each fluctuator contributes independently to the $1 / f$ noise and, therefore, $\alpha$ is a normalized measure for the relative noise of a sample [40]. Importantly, Hooge's law predicts that the noise amplitude is proportional to the bias squared $\left[S_{V}(f) \propto V_{s d}^{2}\right.$ and $\left.S_{I_{s d}}(f) \propto I_{s d}^{2}\right]$ for a sample of constant resistance $R[40,41]$. This bias dependence is also observed here (Fig. 2), confirming that the noise in OECTs is also caused by resistance fluctuations. In other words, the applied source-drain voltage does not generate the noise but allows for measurements of it [40].

To take the scaling with bias into account, we plot [Fig. 3(a)] the normalized current noise $S_{I_{s d}} / I_{s d}^{2}$ versus the source-drain current $I_{s d}$ extracted at a fixed frequency $f=10 \mathrm{~Hz}$, for a $(25 \times 25)-\mu \mathrm{m}$ channel transistor gated to different resistance values ( $V_{s d}$ is kept constant at $100 \mathrm{mV}$ ). To get a first insight into the nature of the noise, we compare the experimental data (the black squares) with two models, commonly used to describe and classify $1 / f$ noise. These two models have emerged from the fact that, although it is accepted that resistance fluctuations are the source of the $1 / f$ noise in electronic devices, its true origin is not fully understood. Since the electrical conductivity $\sigma$ of a semiconductor is generally given by $\sigma=e p \mu$, with $e$ being the elementary charge, $p$ the charge carrier density, and $\mu$ the charge carrier mobility, fluctuations in both $\mu$ and $p$ could lead to the observed noise. Models assuming the former are called mobility-fluctuation models. One important member of this group is the $\alpha$-noise model, which has been widely applied to describe noise in homogeneous samples $[37,40,42]$. It follows directly from Hooge's law assuming that the number of fluctuators $N$ is given by the number of charge carriers $(N=p w l d$, with $p$ being the hole density and wld the volume of the channel). The $\alpha$-noise model reads

$$
\frac{S_{I_{s d}}}{I_{s d}^{2}}=\frac{\alpha}{f p w l d}=\frac{\alpha e \mu V_{s d}}{f I_{s d} l^{2}}
$$

where the right term is obtained using Ohm's law and the expression for the conductivity $\sigma=e p \mu$. In the literature, the $\alpha$-noise model is often called Hooge's law [19,20,27]. However, since the model already assumes a homogeneous sample (which is not given in Hooge's original law), we use here the term $\alpha$-noise model. Importantly, the $\alpha$-noise model predicts $S_{I_{s d}} / I_{s d}^{2} \propto 1 / I_{s d}$ for a constant source-drain voltage, as shown by the green line in Fig. 3(a). Clearly, the $\alpha$-noise model cannot explain our data, and hence mobility fluctuations can be excluded as the origin of the noise in OECTs. It can be argued that the $1 / I_{s d}$ characteristic is valid only if the current depends linearly on the gate voltage. If, however, the current scales exponentially with 


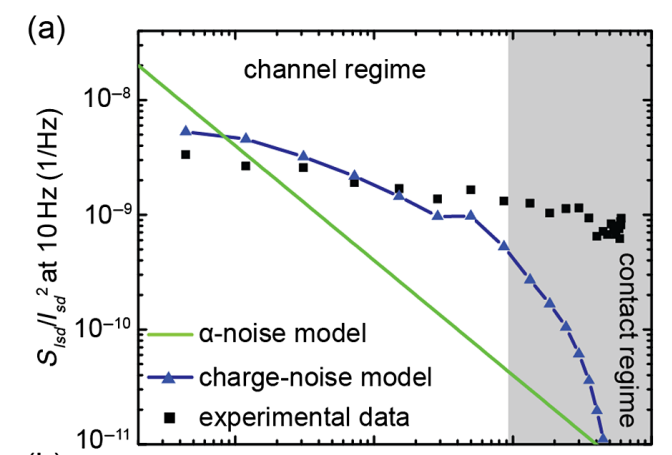

(b)

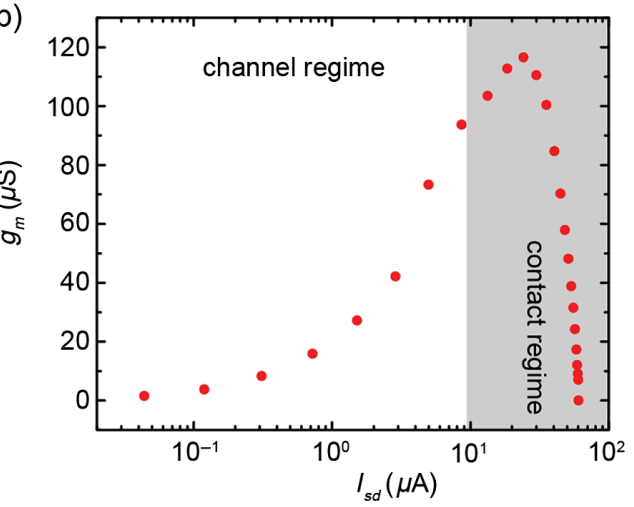

FIG. 3. (a) Normalized source-drain current noise $S_{I_{s d}} / I_{s d}^{2}$ versus the source-drain current $I_{s d}$ at $10 \mathrm{~Hz}$ for a $(25 \times$ 25)- $\mu \mathrm{m}$ OECT biased at $V_{s d}=100 \mathrm{mV}$ (the black symbols). The green solid line shows the prediction following from the $\alpha$ noise model. The blue triangular symbols are calculated using the charge-noise model, which fits the experimental data well up to $I_{s d}=9 \mu \mathrm{A}$. For larger source-drain currents, the data deviate from the charge-noise model due to additional contributions from the contacts (the contact regime). (b) Transconductance $g_{m}$ versus the source-drain current $I_{s d}$.

gate voltage as in the subthreshold regime of the transistor, the slope of the green line should be modified correspondingly [27]. We find also that adapting the slope of the line does not give a satisfactory fit (see Fig. S2 in the Supplemental Material [35]) and, therefore, the $\alpha$-noise model can definitely be excluded.

As an alternative to the $\alpha$-noise model, we apply the charge-noise model [31] shown as the blue fit line in Fig. 3(a). The charge-noise model is a member of the number-fluctuation models which assume that the charge carrier density (in the case of PEDOT:PSS, the hole density) is the fluctuating quantity instead of the mobility [31]. These fluctuations in $p$ can be calculated back to the gate using the gate-referred voltage noise $S_{V_{g}}$, which is connected with the current noise via the transistor transconductance: $S_{I_{s d}}=g_{m}^{2} S_{V_{g}}[20,22,38]$.

The gate-referred voltage noise can be regarded as the noise power of the gate voltage if the transistor channel itself is ideal and noise-free. In typical sensing applications, changes in gate potential $\Delta V_{\mathrm{LG}}$, originating from the adsorption of chemical species, need to be recorded. The gate-referred voltage noise allows for the direct comparison of the noise with the signal of the gate voltage, thereby yielding the signal-to-noise ratio $\mathrm{SNR}=\Delta V_{\mathrm{LG}} / \sqrt{S_{V_{g}}}$ [22]. The charge-noise model was successfully applied to describe $1 / f$ noise measured for liquid-gated SWCNTs $[27,28]$ and single-bilayer graphene $[19,25,26]$ where the channel material is in direct contact with the electrolyte.

The observed $1 / f$ noise is associated with charge fluctuations caused either by trap states in the substrate for substrate-bound SWCNTs [27,28] and graphene devices $[19,25]$ or by the Brownian motion of ions of the electrolyte for suspended carbon nanotubes (CNTs) [28]. Also, the $1 / f$ noise measured for liquid-gated SiNWs follows the charge-noise model, where the noise source is associated with either trapping and/or detrapping at the silicon/oxide interface [20] or fluctuations of the ions in the electrolyte [22]. Independent of the exact noise source, the charge-noise model assumes a charge-noise spectral power density $S_{q}(f)$ in close proximity to the transport material, which couples with an effective gate capacitance $C_{\text {gate }}$ to the device, thereby modulating the carrier density. Consequently, the fluctuating quantity is not the mobility-as in the case of the $\alpha$-noise model—but the number of charge carriers. This model is expressed by

$$
\frac{S_{I_{s d}}}{I_{s d}^{2}}=\frac{g_{m}^{2} S_{V_{g}}}{I_{s d}^{2}}=\frac{g_{m}^{2}}{I_{s d}^{2}} \frac{S_{q}}{C_{\text {gate }}^{2}} .
$$

Measured at a specific frequency, both $S_{q}$ and $C_{\text {gate }}$ are constant for a device with a given geometry; therefore, $S_{V_{g}}=S_{q} / C_{\text {gate }}^{2}$ is also constant [19,31]. Consequently, the charge-noise model predicts $S_{I_{s d}} / I_{s d}^{2} \propto g_{m}^{2} / I_{s d}^{2}$. As shown in Fig. 3(a), the charge-noise model (the blue fit) agrees well with the experimental data for sufficiently small source-drain currents. In this regime, the transistor behavior is fully determined by the properties of the PEDOT:PSS channel, while the influence of the contact resistance is negligible. In the following, we refer to this regime as the channel regime, as indicated in Fig. 3.

The deviation from the model at high source-drain currents is an expected transition from the channel regime to the contact regime highlighted by the gray shaded area in Fig. 3. In the contact regime, the electrode contacts start to contribute significantly to the noise because the resistance of the PEDOT:PSS channel becomes smaller than the contact resistance [43]. The transition between the two regimes is best understood by looking at the transconductance shown in Fig. 3(b), as described previously for SiNWs [20]. When the transconductance approaches its maximum value, the resistance of the channel becomes smaller than the contact resistance, and the noise is mainly determined by the properties of the contacts, rather than the PEDOT: PSS channel. Therefore, we can approximate the position of the regime transition as the value of $I_{s d}$ near the 

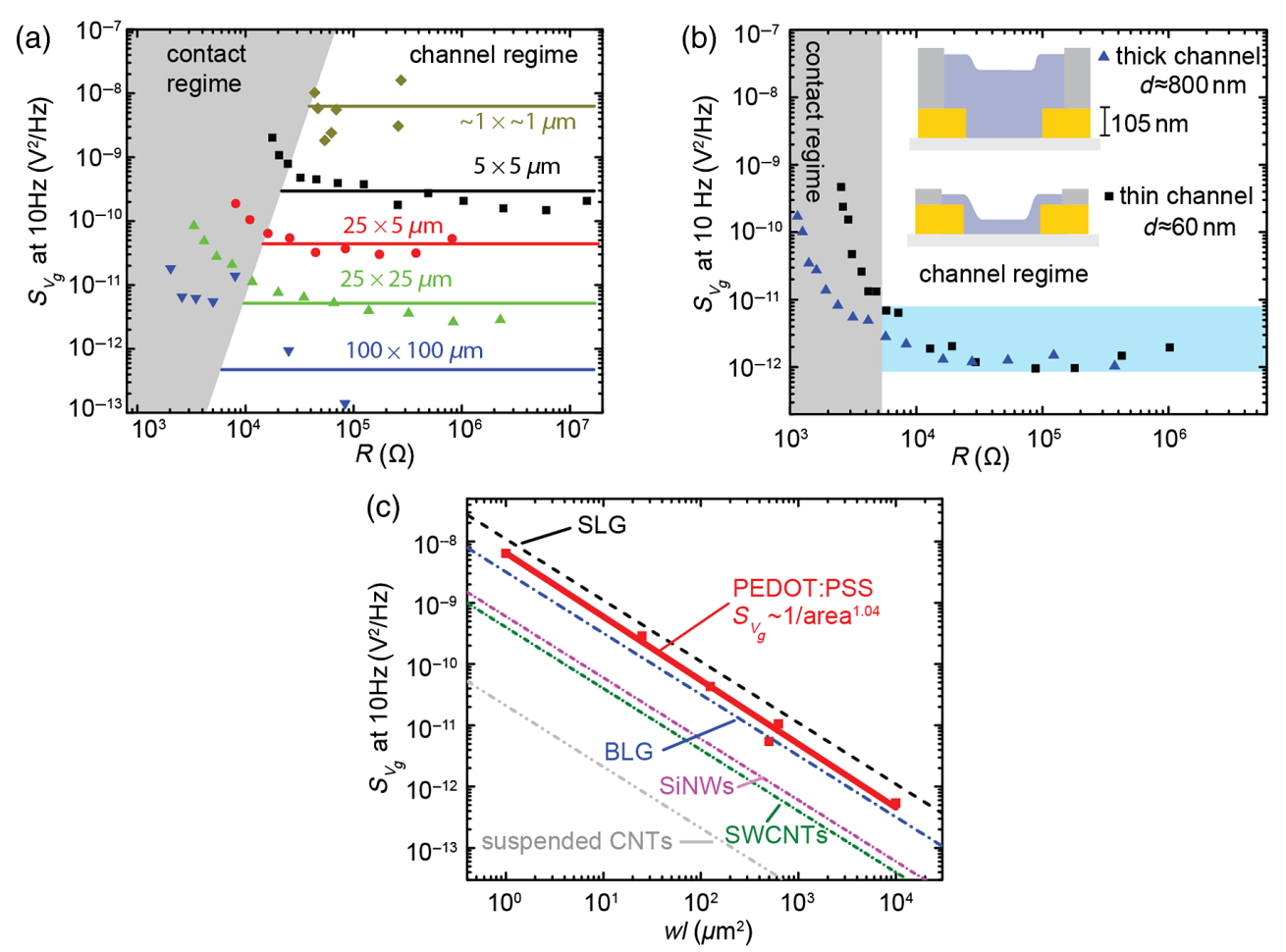

FIG. 4. (a) Gate-referred voltage noise $S_{V_{g}}$ versus the resistance $R$ for five OECTs, with different dimensions as indicated in the graph. At small resistance values, $S_{V_{g}}$ increases due to additional contributions by the contact resistance (contact regime, the gray area). Horizontal lines indicate the average value of $S_{V_{g}}$ for all data points in the channel regime for a particular device. (b) Gate-referred voltage noise $S_{V_{g}}$ versus the resistance $R$ of two devices with different thicknesses $d$ : thick PEDOT:PSS $d \approx 800 \mathrm{~nm}$ and thin PEDOT: PSS $d \approx 60 \mathrm{~nm}$. The light-blue area indicates the scattering of the data. (c) Gate-referred voltage noise $S_{V_{g}}$ versus the channel area $w l$ revealing $S_{V_{g}} \propto 1 / \mathrm{wl}$. The different dashed lines represent the theoretical values obtained for single-layer (SLG) and bilayer graphene (BLG) [19], substrate-bound SWCNTs [27], suspended CNTs [28], and SiNWs [20].

maximum of the transconductance, as indicated in Fig. 3(b).

A more detailed discussion of the contact regime is given in the Supplemental Material [35]. Similar results are also obtained for devices with different geometries (see the Supplemental Material, Fig. S1 [35]). As proposed by Tersoff et al. [31] and Heller et al. [19], the contact noise can be modeled as a noise contribution generated by a gateindependent series resistance to also fit the data in the contact regime. Although this correction improves the fit for large source-drain currents (see the Supplemental Material, Fig. S3 [35]), we focus on the channel regime because the best SNR is achieved only for sufficiently small source-drain currents, as we show in the following. Furthermore, to compare different channel materials, we use the value of the gate-referred voltage noise, which is constant only in the channel regime.

Our measurements suggest that the low-frequency $1 / f$ noise in OECTs follows a charge-noise model similar to that proposed for graphene [19] and CNT [27] transistors. However, in strong contrast to these devices, where a welldefined, sharp interface exists between electrolyte and active material, in OECTs, the electrolyte penetrates into the conducting polymer channel. This unique property raises a question about the origin of the noise in OECTs, which we discuss in the following by studying the noise scaling with geometry.

\section{B. Noise scaling with geometry}

Figure 4(a) shows the gate-referred voltage noise $S_{V_{g}}$ versus the resistance $R$ at $10 \mathrm{~Hz}$ for devices with varying channel widths and lengths but a constant film thickness $(d \approx 110 \mathrm{~nm})$. We find $S_{V_{g}}$ to be independent of the resistance value as long as the transistor is operated in the channel regime, in agreement with the charge-noise model. As soon as the contacts also contribute to the noise, at low-resistance values, the gate-referred noise increases drastically, as observed in Fig. 4(a). The transition between the contact regime and the channel regime has been determined to be the value of the channel resistance around the maximum transconductance, where the charge-noise model starts to deviate considerably from the experimental data. As the contact resistance is expected to scale with the inverse channel width $w$, the contact regime shifts towards lower resistance values on the horizontal axis for an 
increasing $w$, as highlighted by the gray area. We observe that the gate-referred voltage noise decreases for increasing the channel area indicated by the horizontal lines in Fig. 4(a). We further investigate the scaling of the noise with channel volume, measuring samples with two different thicknesses, $d^{\text {thin }} \approx 60 \mathrm{~nm}$ and $d^{\text {thick }} \approx 800 \mathrm{~nm}$, but the same width and length (5 and $100 \mu \mathrm{m}$, respectively). Figure 4(b) shows $S_{V_{g}}$ versus $R$ for the two types of devices. As expected from the device geometry, the thick sample exhibits both lower channel and lower contact resistance (see the Supplemental Material, Fig. S6 [35]). Therefore, we find a lower noise for the thick PEDOT:PSS in the contact regime. When operating the transistor in accumulation, thick OECTs are preferred, in agreement with a recent publication of Rivnay et al. [9]. However, in the channel regime, we observe no significant difference in noise for the two thicknesses. It means that for achieving the best SNR, thin OECTs are as good as their thicker counterparts.

The observed dependence of $S_{V_{g}}$ on the channel areabut not on the channel thickness-needs further discussion. Since $S_{V_{g}}=S_{q} / C_{\text {gate }}^{2}$, the scaling of $S_{V_{g}}$ with geometry depends on the corresponding scaling of the charge noise $S_{q}$ and the gate capacitance $C_{\text {gate }}$. Our experimental data could be explained by assuming that both $C_{\text {gate }}$ and $S_{q}$ scale only with the area leading to $S_{V_{g}} \propto 1 / w l$, as is commonly accepted for liquid-gated CNTs and graphene transistors [19,25-28]. However, the absence of a clear interface between the polymer channel and the electrolyte might lead to a different geometry scaling of $S_{V_{g}}$. For example, it has recently been demonstrated that the gating process of PEDOT:PSS OECTs involves the whole channel volume due to the ionic permeability of the polymer. Therefore, both transconductance and effective gate capacitance depend on the channel thickness leading to $g_{m} \propto w d / l$ and $C_{\text {gate }} \propto$ wld [9]. Consequently, the noise might originate from doping and/or dedoping processes within the bulk of the material, where the Brownian motion of cations leads to a volume-dependent noise source $S_{q}(f)=S_{q}^{*} w l d / f$, with $S_{q}^{*}$ representing the charge-noise power density per volume. Although not clearly observed here, the unique volumetric gating behavior of OECTs makes a volume-dependent noise source $S_{q}$ plausible. Independent of the exact noise mechanism, the chargenoise model predicts $S_{V_{g}} \propto 1 / w l$, which we verify experimentally in the following.

Figure 4(c) shows the value of the plateau of the gatereferred voltage noise of Fig. 4(a) versus the channel area $w l$, measured for devices of the constant thickness $d \approx 110 \mathrm{~nm}$. The thickness $d \approx 110 \mathrm{~nm}$ represents a practical trade-off between high transconductance and fast response times [7,9]. As proposed by the charge-noise model, we indeed find that the gate-referred voltage noise scales with $S_{V_{g}} \propto 1 / w l$. Furthermore, we compare the value of the gate-referred voltage noise with values obtained in the literature for transistors based on singleand bilayer graphene [19], SiNWs [20], substrate-bound SWCNTs [27], and suspended CNTs [28], represented by the dashed colored lines in Fig. 4(c) $\left(S_{V_{g}}\right.$ for graphene and SWCNTs measured at $1 \mathrm{~Hz}$ has been converted to $S_{V_{g}}$ at $10 \mathrm{~Hz}$ for reasons of comparison). Interestingly, we find that the noise of OECTs is comparable to graphene devices, while it is slightly higher compared to SiNWs and substrate-bound SWCNTs transistors. Suspending the CNTs has been shown to significantly reduce the noise, an indication that the charge noise caused by the substrate is significantly contributing to the $1 / f$ noise [28]. The gatereferred voltage noise allows us to calculate the corresponding charge noise, $S_{q}$, which can be compared with state-of-the-art inorganic transistor devices, particularly socalled single-electron transistors (SETs) that are used for metrology due to their optimized, strongly reduced noise characteristics. Assuming a volume-dependent capacitance $C^{*}=39.3 \mathrm{~F} / \mathrm{cm}^{3}$ [9], we estimate $\sqrt{S_{q}}=\sqrt{C^{2} S_{V_{g}}} \approx$ $8.54 \times 10^{-15} \mathrm{C} / \sqrt{\mathrm{Hz}} \approx 5.34 \times 10^{4} \mathrm{e} / \sqrt{\mathrm{Hz}}$, with $C=$ $C^{*} V=2.7 \mathrm{nF}$ being the capacitance of the device shown in Figs. 1 and 3 with the volume $V=$ wld $(w=25 \mu \mathrm{m}, l=25 \mu \mathrm{m}$, and $d=110 \mathrm{~nm})$, with $S_{V_{g}}=$ $1 \times 10^{-11} \mathrm{~V}^{2} / \mathrm{Hz}$ for a $(25 \times 25)-\mu \mathrm{m}$ OECT, as given by the fit line in Fig. 4(c). Compared to the SET devices presented in the literature $[23,32,33]$, our $\sqrt{S_{q}}$ is much larger, which is not surprising given the large capacitance and volume of the OECTs.

\section{Signal-to-noise ratio and limit of detection}

To evaluate the resulting SNR for the studied devices, we finally compare $S_{V_{g}}$ with typical signals of OECT biosensors. Recently, PEDOT:PSS-based OECTs have been modified with ion-sensitive membranes to achieve a potassium- $\left(\mathrm{K}^{+}-\right)$selective sensor [13]. Typically, the interfacial potential difference (also called the membrane voltage) $V_{m}$ of such ion-sensitive membranes changes according to the Nernst equation by $\Delta V_{m}=59.5 \mathrm{mV}$ per decade in concentration $(\mathrm{mV} / \mathrm{dec})$ of the ion of interest. $V_{m}$ thereby acts as an additional gating signal modulating the current of the transistor $\left(\Delta V_{\mathrm{LG}}=\Delta V_{m}\right)$.

Since the signal originates at the gate of the transistor, it has to be compared with the noise at the gate, given by the gate-referred voltage noise $S_{V_{g}}$. We consider a typical OECT $[w=l=25 \mu \mathrm{m}, d \approx 110 \mathrm{~nm}$, as shown in Fig. 4(a), the green triangles] modified with a membrane that exhibits a Nernstian response to potassium ions over a concentration range from $1 \mu M$ to $10 \mathrm{~m} M$. With this system, an increase in potassium concentration by one order of magnitude can be detected with $\mathrm{SNR}=\Delta V_{m} / \sqrt{S_{V_{g}}} \approx$ $18816 / \sqrt{\mathrm{Hz}}$ at $10 \mathrm{~Hz}$, with a $1-\mathrm{Hz}$ bandwidth. This result 
is obtained by using $\Delta V_{m}=59.5 \mathrm{mV}$ and $S_{V_{g}}=$ $1 \times 10^{-11} \mathrm{~V}^{2} / \mathrm{Hz}$ [using again the value of $S_{V_{g}}$ for a $(25 \times$ 25) $-\mu \mathrm{m}$ OECT, as given by the fit line in Fig. 4(c)].

The smallest detectable voltage change $\Delta V_{m \text {,min }}$ is given by $S_{V_{g}}: \Delta V_{m, \text { min }}=\sqrt{S_{V_{g}}} \approx 3.2 \times 10^{-6} \mathrm{~V} / \sqrt{\mathrm{Hz}}$ for the OECT. In other words, $\Delta V_{m \text {,min }}$ can still be detected with $\mathrm{SNR}=1$ at $10 \mathrm{~Hz}$ and with a $1-\mathrm{Hz}$ bandwidth. This value corresponds to $0.0053 \%$ of a full Nernstian shift of $59.5 \mathrm{mV} / \mathrm{dec}$ of $\mathrm{K}^{+}$, independent of the concentration range, as long as the membrane exhibits a Nernstian response. However, the corresponding LOD depends on the actual background concentration. This relation is evident from the fact that $0.0053 \%$ of a Nernstian shift corresponds to a higher absolute change in concentration at $1 \mathrm{~m} M$ than at $1 \mu M$.

Formally, we define here the LOD as $\Delta c_{\mathrm{K}^{+}, \min }=$ $c_{\mathrm{K}^{+}, \min }-c_{\mathrm{K}^{+}}$, where $c_{\mathrm{K}^{+} \text {,min }}$ is the smallest detectable absolute concentration and $c_{\mathrm{K}^{+}}$is the background concentration. $c_{\mathrm{K}^{+}, \text {min }}$ depends on the SNR and the background concentration given by

$$
\log \left(c_{\mathrm{K}^{+}, \min } / c_{0}\right)=\mathrm{SNR}^{-1}+\log \left(c_{\mathrm{K}^{+}} / c_{0}\right),
$$

where $c_{0}=1 M$ is the concentration of the standard state commonly used to make the argument of the logarithm dimensionless (the normalized concentration is then usually called the chemical activity). With $\mathrm{SNR}=18816$ and $c_{\mathrm{K}^{+}}=1 \mu M$, this calculation leads to $c_{\mathrm{K}^{+}, \min }=$ $1.000122 \mu M$. This result indicates that an increase $\Delta c_{\mathrm{K}^{+}, \text {min }}=c_{\mathrm{K}^{+}, \text {min }}-c_{\mathrm{K}^{+}}=122 \mathrm{p} M$ (LOD) can still be detected with $\mathrm{SNR}=1$ at $10 \mathrm{~Hz}$, with a $1-\mathrm{Hz}$ bandwidth at a background concentration of $c_{\mathrm{K}^{+}}=1 \mu M$, using a $(25 \times 25)-\mu \mathrm{m}$ OECT modified with a potassium-selective membrane with a Nernstian response between $1 \mu M$ and $10 \mathrm{~m} M$. Further details are given in the Supplemental Material [35].

\section{CONCLUSIONS}

In this work, we study the low-frequency $1 / f$ noise in PEDOT:PSS-based OECTs. We are able to quantify the SNR and the LOD of an OECT in a typical ion-sensing measurement. A comparison with the literature shows that the noise of PEDOT:PSS OECTs with a typical thickness of $d \approx 110 \mathrm{~nm}$ is comparable to graphene transistors and only slightly higher than SiNWs and substrate-bound SWCNT transistors. The experimental data are in good agreement with the charge-noise model, which reveals that the noise is caused by charge fluctuations in the proximity of the channel or within the active material. Our results provide an additional design rule for the application of OECTs in biochemical and electrostatic sensing experiments, recommending the use of large-area polymer channels to maximize the SNR.

\section{ACKNOWLEDGMENTS}

The authors gratefully acknowledge the support by the European Commission under the FP7-NMP project Hysens (Contract No. 263091), the European Commission under the FP7-ICT project Symone (Contract No. 105244), the H2020 FET Open project RECORD-IT (Contract No. 664786), and the Swiss National Science Foundation as part of the NCCR Molecular Systems Engineering. M.S. acknowledges support from the I edition of the BBVA Foundation Grants for Researchers and Cultural Creators. The authors further acknowledge financial support from the Spanish Ministry of Economy and Competitiveness (MINECO) via the Unidad de Excelencia María de Maeztu MDM-2015-0538. The authors would like to thank Marc Ferro and George G. Malliaras (Centre Microélectronique de Provence) for the fruitful discussions.

[1] G. Malliaras and R. Friend, An organic electronics primer, Phys. Today 58, No. 5, 53 (2005).

[2] M. Berggren and A. Richter-Dahlfors, Organic bioelectronics, Adv. Mater. 19, 3201 (2007).

[3] P. Lin and F. Yan, Organic thin-film transistors for chemical and biological sensing, Adv. Mater. 24, 34 (2012).

[4] J. Rivnay, R. M. Owens, and G. G. Malliaras, The rise of organic bioelectronics, Chem. Mater. 26, 679 (2014).

[5] X. Cui, V. A. Lee, Y. Raphael, J. A. Wiler, J. F. Hetke, D. J. Anderson, and D.C. Martin, Surface modification of neural recording electrodes with conducting polymer/ biomolecule blends, J. Biomed. Mater. Res. 56, 261 (2001).

[6] H. S. White, G. P. Kittlesen, and M. S. Wrighton, Chemical derivatization of an array of three gold microelectrodes with polypyrrole: Fabrication of a molecule-based transistor, J. Am. Chem. Soc. 106, 5375 (1984).

[7] D. Khodagholy, J. Rivnay, M. Sessolo, M. Gurfinkel, P. Leleux, L. H. Jimison, E. Stavrinidou, T. Hervé, S. Sanaur, R. M. Owens, and G. G. Malliaras, High transconductance organic electrochemical transistors, Nat. Commun. 4, 2133 (2013).

[8] J. Rivnay, P. Leleux, M. Sessolo, D. Khodagholy, T. Hervé, M. Fiocchi, and G. G. Malliaras, Organic electrochemical transistors with maximum transconductance at zero gate bias, Adv. Mater. 25, 7010 (2013).

[9] J. Rivnay, P. Leleux, M. Ferro, M. Sessolo, A. Williamson, D. A. Koutsouras, D. Khodagholy, M. Ramuz, X. Strakosas, R. M. Owens, C. Benar, J.-M. Badier, C. Bernard, and G. G. Malliaras, High-performance transistors for bioelectronics through tuning of channel thickness, Sci. Adv. 1, e1400251 (2015).

[10] D. A. Bernards, D. J. Macaya, M. Nikolou, J. A. DeFranco, S. Takamatsu, and G. G. Malliaras, Enzymatic sensing with organic electrochemical transistors, J. Mater. Chem. 18, 116 (2008).

[11] L. Kergoat, B. Piro, D. T. Simon, M.-C. Pham, V. Noël, and M. Berggren, Detection of glutamate and acetylcholine with 
organic electrochemical transistors based on conducting polymer/platinum nanoparticle composites, Adv. Mater. 26, 5658 (2014).

[12] C. Liao, C. Mak, M. Zhang, H. L. W. Chan, and F. Yan, Flexible organic electrochemical transistors for highly selective enzyme biosensors and used for saliva testing, Adv. Mater. 27, 676 (2015).

[13] M. Sessolo, J. Rivnay, E. Bandiello, G. G. Malliaras, and H. J. Bolink, Ion-selective organic electrochemical transistors, Adv. Mater. 26, 4803 (2014).

[14] P. Lin, F. Yan, and H. L. W. Chan, Ion-sensitive properties of organic electrochemical transistors, ACS Appl. Mater. Interfaces 2, 1637 (2010).

[15] L. H. Jimison, S. A. Tria, D. Khodagholy, M. Gurfinkel, E. Lanzarini, A. Hama, G. G. Malliaras, and R. M. Owens, Measurement of barrier tissue integrity with an organic electrochemical transistor, Adv. Mater. 24, 5919 (2012).

[16] A. Campana, T. Cramer, D. T. Simon, M. Berggren, and F. Biscarini, Electrocardiographic recording with conformable organic electrochemical transistor fabricated on resorbable bioscaffold, Adv. Mater. 26, 3874 (2014).

[17] X. Strakosas, M. Bongo, and R. M. Owens, The organic electrochemical transistor for biological applications, J. Appl. Polym. Sci. 132, 41735 (2015).

[18] P. Leleux, J. Rivnay, T. Lonjaret, J.-M. Badier, C. Bénar, T. Hervé, P. Chauvel, and G. G. Malliaras, Organic electrochemical transistors for clinical applications, Adv. Healthcare Mater. 4, 142 (2015).

[19] I. Heller, S. Chatoor, J. Männik, M. A. G. Zevenbergen, J. B. Oostinga, A. F. Morpurgo, C. Dekker, and S. G. Lemay, Charge noise in graphene transistors, Nano Lett. 10, 1563 (2010).

[20] K. Bedner, V. A. Guzenko, A. Tarasov, M. Wipf, R. L. Stoop, S. Rigante, J. Brunner, W. Fu, C. David, M. Calame, J. Gobrecht, and C. Schönenberger, Investigation of the dominant $1 / f$ noise source in silicon nanowire sensors, Sens. Actuators B 191, 270 (2014).

[21] X. Duan, Y. Li, N. K. Rajan, D. A. Routenberg, Y. Modis, and M. A. Reed, Quantification of the affinities and kinetics of protein interactions using silicon nanowire biosensors, Nat. Nanotechnol. 7, 401 (2012).

[22] N. K. Rajan, K. Brower, X. Duan, and M. A. Reed, Limit of detection of field effect transistor biosensors: Effects of surface modification and size dependence, Appl. Phys. Lett. 104, 084106 (2014).

[23] N. Clément, K. Nishiguchi, J. F. Dufreche, D. Guerin, A. Fujiwara, and D. Vuillaume, A silicon nanowire ionsensitive field-effect transistor with elementary charge sensitivity, Appl. Phys. Lett. 98, 014104 (2011).

[24] M. V. Hauf, L. H. Hess, J. Howgate, M. Dankerl, M. Stutzmann, and J.A. Garrido, Low-frequency noise in diamond solution-gated field effect transistors, Appl. Phys. Lett. 97, 093504 (2010).

[25] Z. Cheng, Q. Li, Z. Li, Q. Zhou, and Y. Fang, Suspended graphene sensors with improved signal and reduced noise, Nano Lett. 10, 1864 (2010).

[26] Z. Cheng, J. Hou, Q. Zhou, T. Li, H. Li, L. Yang, K. Jiang, C. Wang, Y. Li, and Y. Fang, Sensitivity limits and scaling of bioelectronic graphene transducers, Nano Lett. 13, 2902 (2013).
[27] J. Männik, I. Heller, A. M. Janssens, S. G. Lemay, and C. Dekker, Charge noise in liquid-gated single-wall carbon nanotube transistors, Nano Lett. 8, 685 (2008).

[28] T. Sharf, J. W. Kevek, T. DeBorde, J. L. Wardini, and E. D. Minot, Origins of charge noise in carbon nanotube field-effect transistor biosensors, Nano Lett. 12, 6380 (2012).

[29] C. Bonavolontà, C. Albonetti, M. Barra, and M. Valentino, Electrical mobility in organic thin-film transistors determined by noise spectroscopy, J. Appl. Phys. 110, 093716 (2011).

[30] C. Y. Han, L. X. Qian, C. H. Leung, C. M. Che, and P. T. Lai, A low-frequency noise model with carrier generation-recombination process for pentacene organic thin-film transistor, J. Appl. Phys. 114, 044503 (2013).

[31] J. Tersoff, Low-frequency noise in nanoscale ballistic transistors, Nano Lett. 7, 194 (2007).

[32] A. B. Zorin, F.-J. Ahlers, J. Niemeyer, T. Weimann, H. Wolf, V. A. Krupenin, and S. V. Lotkhov, Background charge noise in metallic single-electron tunneling devices, Phys. Rev. B 53, 13682 (1996).

[33] V. A. Krupenin, D. E. Presnov, M. N. Savvateev, H. Scherer, A. B. Zorin, and J. Niemeyer, Noise in Al single electron transistors of stacked design, J. Appl. Phys. 84, 3212 (1998).

[34] M. Sessolo, D. Khodagholy, J. Rivnay, F. Maddalena, M. Gleyzes, E. Steidl, B. Buisson, and G. G. Malliaras, Easy-to-fabricate conducting polymer microelectrode arrays, Adv. Mater. 25, 2135 (2013).

[35] See Supplemental Material at http://link.aps.org/ supplemental/10.1103/PhysRevApplied.7.014009 for details on the noise data of additional devices, fits using a modified $\alpha$-noise model, fits using the augmented chargenoise model, motivation of the contact regime, transfer characteristics for devices with two different channel thicknesses, details on the calculation of the limit of detection, details on the sample fabrication, and $I-V$ characteristics of OECTs with dimensions approximately equal to $1 \mu \mathrm{m}$.

[36] D. A. Bernards and G. G. Malliaras, Steady-state and transient behavior of organic electrochemical transistors, Adv. Funct. Mater. 17, 3538 (2007).

[37] F. N. Hooge, $l / f$ noise sources, IEEE Trans. Electron Devices 41, 1926 (1994).

[38] M. von Haartman and M. Östling, Low-Frequency Noise in Advanced MOS Devices, Analog Circuits and Signal Processing (Springer, New York, 2007).

[39] P. Dutta and P. M. Horn, Low-frequency fluctuations in solids: 1/ $f$ noise, Rev. Mod. Phys. 53, 497 (1981).

[40] F. N. Hooge, T. G. M. Kleinpenning, and L. K. J. Vandamme, Experimental studies on $1 / f$ noise, Rep. Prog. Phys. 44, 479 (1981).

[41] M. B. Weissman, $1 / f$ noise and other slow, nonexponential kinetics in condensed matter, Rev. Mod. Phys. 60, 537 (1988).

[42] L. K. J. Vandamme, X. Li, and D. Rigaud, $1 / f$ noise in MOS devices, mobility or number fluctuations?, IEEE Trans. Electron Devices 41, 1936 (1994).

[43] V. Kaphle, S. Liu, A. Al-Shadeedi, C.-M. Keum, and B. Lüssem, Contact resistance effects in highly doped organic electrochemical transistors, Adv. Mater. 28, 8766 (2016). 